\title{
On the inverse Magnus effect for flow past a rotating cylinder
}

\author{
Benzi John, Xiao-Jun Gu, Robert W. Barber and \\ David R. Emerson
}

\section{Published version information}

Citation: B John et al. "On the inverse Magnus effect for flow past a rotating cylinder." AIP Conference Proceedings, vol. 1786 (2016): 050022. Is in proceedings of: 30th International Symposium on Rarefied Gas Dynamics (RDG 30), Victoria, British Columbia, Canada, 10-15 Jul 2016.

DOI: $\underline{10.1063 / 1.4967572}$

This article may be downloaded for personal use only. Any other use requires prior permission of the author and AIP Publishing.

This version is made available in accordance with publisher policies. Please cite only the published version using the reference above. 


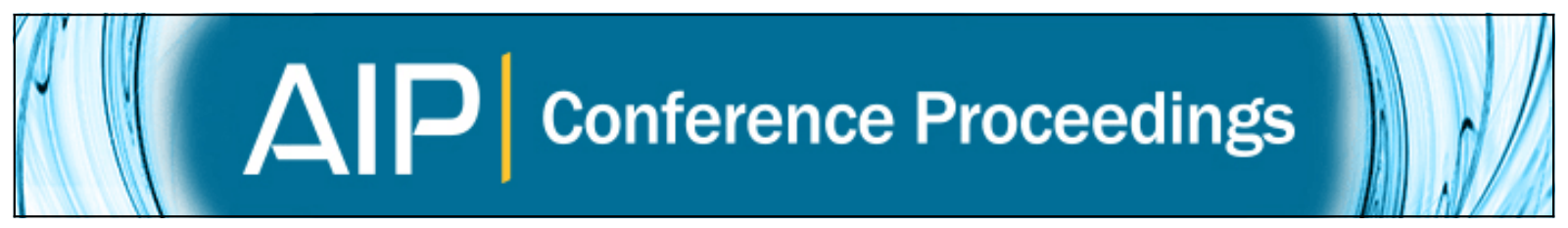

\section{On the inverse Magnus effect for flow past a rotating cylinder}

Benzi John, Xiao-Jun Gu, Robert W. Barber, and David R. Emerson

Citation: AIP Conference Proceedings 1786, 050022 (2016); doi: 10.1063/1.4967572

View online: http://dx.doi.org/10.1063/1.4967572

View Table of Contents: http://scitation.aip.org/content/aip/proceeding/aipcp/1786?ver=pdfcov

Published by the AIP Publishing

Articles you may be interested in

Flow past two rotating cylinders

Phys. Fluids 23, 014102 (2011); 10.1063/1.3528260

On the inverse Magnus effect in free molecular flow

Phys. Fluids 16, L9 (2004); 10.1063/1.1633265

Laminar flow past a rotating circular cylinder

Phys. Fluids 11, 3312 (1999); 10.1063/1.870190

Rotating flow past a sliced cylinder

Phys. Fluids 8, 131 (1996); 10.1063/1.868821

Rotating oscillatory flow past a cylinder

Phys. Fluids A 5, 868 (1993); 10.1063/1.858633 


\title{
On the Inverse Magnus Effect for Flow Past a Rotating Cylinder
}

\author{
Benzi John, Xiao-Jun Gu, Robert W. Barber and David R. Emerson ${ }^{\text {a) }}$ \\ Scientific Computing Department, STFC Daresbury Laboratory, Warrington, WA4 4AD, UK \\ ${ }^{\text {a)} C o r r e s p o n d i n g ~ a u t h o r: ~ d a v i d . e m e r s o n @ s t f c . a c . u k ~}$
}

\begin{abstract}
Flow past a rotating cylinder has been investigated using the direct simulation Monte Carlo method. The study focuses on the occurrence of the inverse Magnus effect under subsonic flow conditions. In particular, the variations in the coefficients of lift and drag have been investigated as a function of the Knudsen and Reynolds numbers. Additionally, a temperature sensitivity study has been carried out to assess the influence of the wall temperature on the computed aerodynamic coefficients. It has been found that both the Reynolds number and the cylinder wall temperature significantly affect the drag as well as the onset of lift inversion in the transition flow regime.
\end{abstract}

\section{INTRODUCTION}

The Magnus effect, where a rotating body experiences a transverse aerodynamic lift force, has been investigated by many researchers [1-3]. The pioneering work investigating the lift force on rotating bodies is attributed to Benjamin Robins [4] in the eighteenth century and Gustav Magnus [5] in the nineteenth century. However, the origin of such studies can be traced back as early as the seventeenth century to Sir Isaac Newton [6]. Nowadays, numerous practical applications of the Magnus effect can be found in a wide range of maritime and aeronautical devices $[7,8]$.

In the case of a rotating cylinder, the Magnus effect is often explained in terms of potential flow theory using the superposition of a uniform flow field, a doublet, and a potential (or free) vortex [1]. However, this classical hydrodynamics description of the Magnus effect is a simplified explanation due to the absence of any boundary layers on the cylinder. In reality, the flow around a rotating circular cylinder is far more complex than that obtained using simple potential theory. Swanson [3] was the first to explain the Magnus effect in terms of the unsymmetrical flow patterns caused by the upper and lower boundary layers separating at different relative positions around the circumference of the rotating cylinder. Experimental evidence shows that the magnitude of the Magnus lift force depends on many factors including the rotational and freestream speeds, the Reynolds and Mach numbers, and the surface roughness of the cylinder.

Although there have been many studies of the Magnus effect in the continuum flow regime $[3,9,10]$, relatively few studies have considered rarefied conditions. Of particular interest is the lift generated in the free molecular limit which has been shown analytically to be in the opposite direction to the conventional Magnus force [11-15]. The reversal in the direction of the lift force is usually referred to as the inverse Magnus effect. Apart from analytical studies in the free-molecular limit, there has been comparatively little work covering the entire Knudsen regime. Riabov [16] used the direct simulation Monte Carlo (DSMC) method [17] to investigate the lift and drag coefficients on a rotating circular cylinder for a range of Knudsen numbers and roll parameters. More recently, the authors [18, 19] have conducted comprehensive DSMC studies of the onset of the inverse Magnus effect. Our previous work has focused specifically on high-speed flow conditions in the transonic and supersonic regimes, and has shown that there 
is a subtle interplay between the Mach number and the Knudsen number for the occurrence of the inverse Magnus effect. In the present paper, our numerical investigations concentrate on subsonic flow past a rotating cylinder at low Reynolds numbers. The study investigates the aerodynamic forces acting on a rotating cylinder in the transition flow regime and examines the role of the Reynolds number and Knudsen number on the lift inversion phenomenon. Only the transition regime has been considered since DSMC computations in the early slip flow regime are computationally prohibitive, especially for low-speed flows [20]. A temperature sensitivity study has also been carried out to assess the influence of the temperature of the cylinder on the lift inversion phenomenon.

\section{DSMC CODE AND PROBLEM FORMULATION}

The DSMC simulations have been performed using dsmcFoam [21] which operates within the framework of the open-source code, OpenFOAM [22]. The dsmcFoam C++ library has previously been validated against a variety of benchmark test cases involving a wide range of flow conditions [23, 24]. Additional validation tests have been presented by the authors [19] comparing the computed drag coefficient for flow past a stationary (non-rotating) cylinder against experimental data [25] and the analytical free-molecular solution [26].

We consider a right circular cylinder rotating in the counter-clockwise direction with its axis perpendicular to the flow direction, as shown schematically in Fig. 1. For this particular configuration, with the flow impinging on the cylinder in the positive $x$-direction, the conventional Magnus lift force acts in the negative $y$-direction. The speed of rotation of the cylinder is characterized in terms of the rotation parameter, $W$, which is defined as $W=\omega R / U_{\infty}$, where $\omega$ is the angular velocity, $R$ is the radius of the cylinder and $U_{\infty}$ is the freestream velocity. In the present study, a constant rotation parameter of $W=2$ is considered and the gas-surface interactions at the wall of the cylinder are assumed to be fully diffuse. Unless otherwise specified, the cylinder wall temperature is assumed to be equal to the freestream temperature, i.e. $T_{w}=T_{\infty}$. The simulations are performed using a freestream temperature of $T_{\infty}=273 \mathrm{~K}$ whilst the variation in the Knudsen number is achieved by adjusting the freestream pressure, $p_{\infty}$.

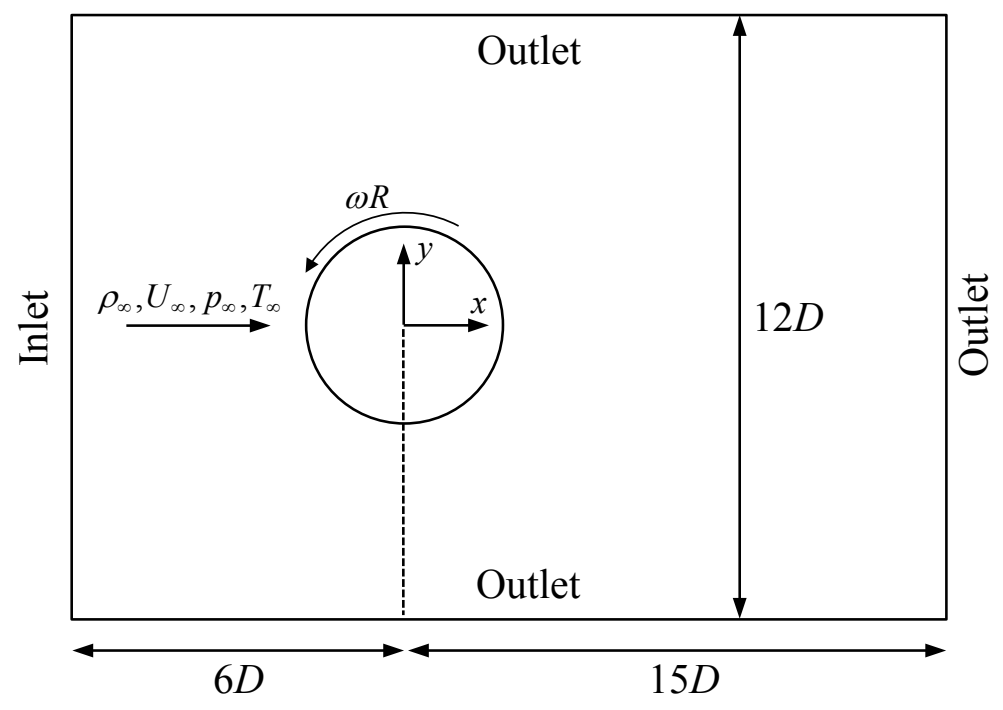

FIGURE 1. Schematic of computational domain (not to scale)

The computational domain consists of a rectangular region with the origin located at the center of the cylinder, as illustrated in Fig. 1. The upstream left-hand boundary is treated as an inlet whereas the other boundaries are treated as outlets. The boundary conditions at the inflow and outflow are implemented by injecting particles with a Maxwellian velocity distribution corresponding to the physical conditions at the boundary [17]. The subsonic outflow boundaries employ a characteristic boundary condition [19, 27-28]; this involves specifying the exit pressure while other flow variables such as the velocity and density are derived from extrapolated values from the adjacent cells inside the computational domain. A preliminary study was undertaken to investigate the effect of the 
size of the computational domain. Accordingly, the inflow boundary was located a distance of $6 D$ upstream of the cylinder while the outflow boundary was located a distance of $15 D$ downstream of the cylinder, where $D$ is the diameter of the cylinder. The predicted lift and drag coefficients for this particular domain were found to be in very good agreement with those obtained from a much larger domain.

The DSMC simulations employ a variable hard sphere model corresponding to the argon atom. We have implemented the usual recommendations for the cell size, time step, and particle numbers [17, 20, 29, 30]. The cell sizes are chosen to be smaller than one-third of the freestream mean free path, $\lambda_{\infty}$, while the time step is selected to be five times smaller than $\Delta x_{\min } /\left(V_{m p}+U_{\infty}+U_{w}\right)$, where $\Delta x_{\min }$ is the smallest cell dimension, $V_{m p}$ is the most probable molecular velocity given by $V_{m p}=\left(2 \mathcal{R} T_{\infty}\right)^{1 / 2}, U_{\infty}$ is the freestream velocity, $U_{w}$ is the tangential wall velocity of the cylinder and $\mathcal{R}$ is the specific gas constant. The freestream mean free path can be obtained from $\lambda_{\infty}=\left(\mu_{\infty} / p_{\infty}\right)\left(\pi \mathcal{R} T_{\infty} / 2\right)^{1 / 2}$ where $p_{\infty}$ is the freestream pressure and $\mu_{\infty}$ is the coefficient of dynamic viscosity at the freestream temperature, $T_{\infty}$. To minimize statistical noise, an average of at least 100 particles per cell has been used, and the sampling phase has been carried out over a period of several hundred thousand time steps. To reduce the computational cost of the simulations, the numerical study has been carried out in two dimensions. The simulations typically employ between $\sim 6,000$ and $\sim 10,000$ computational cells and between $\sim 600,000$ and $\sim 1$ million particles.

The flow conditions can be characterized in terms of the Knudsen, Reynolds and Mach numbers. The Knudsen and Reynolds numbers are based on the diameter of the cylinder and the freestream conditions, i.e. $K n=\lambda_{\infty} / D$ and $R e=\rho_{\infty} U_{\infty} D / \mu_{\infty}$ where $\lambda_{\infty}$ is the freestream mean free path and $\rho_{\infty}$ is the freestream density. The Mach number is defined as $M a=U_{\infty} /\left(\gamma \mathcal{R} T_{\infty}\right)^{1 / 2}$ where $\gamma$ is the adiabatic index (i.e. the ratio of the specific heat at constant pressure and the specific heat at constant volume). In the present study, we consider Knudsen numbers in the range, $0.3 \leq K n \leq 1$ and Reynolds numbers in the range, $0.25 \leq R e \leq 1$. The corresponding Mach number lies between $0.08 \leq M a \leq 0.6$. As detailed by Schaaf and Chambré [26], for low Reynolds number flows $(i . e$. $R e<1)$, the Knudsen, Reynolds and Mach numbers are interrelated via $K n \sim M a / R e$. In the following sections, we have chosen to present the results in terms of the Reynolds number and the Knudsen number.

\section{RESULTS AND DISCUSSION}

The variations in the lift and drag coefficients with Knudsen number are shown in Fig. 2 for four different Reynolds numbers $(R e=0.25,0.5,0.75$ and 1$)$. The lift coefficient is defined as $C_{L}=F_{L} /\left(1 / 2 \rho_{\infty} A U_{\infty}{ }^{2}\right)$ where $F_{L}$ is the total lift force acting on the cylinder in the $y$-direction and $A$ is the projected area of the cylinder normal to the flow. Similarly, the drag coefficient is defined as $C_{D}=F_{D} /\left(1 / 2 \rho_{\infty} A U_{\infty}{ }^{2}\right)$ where $F_{D}$ is the total drag force on the cylinder in the $x$-direction.

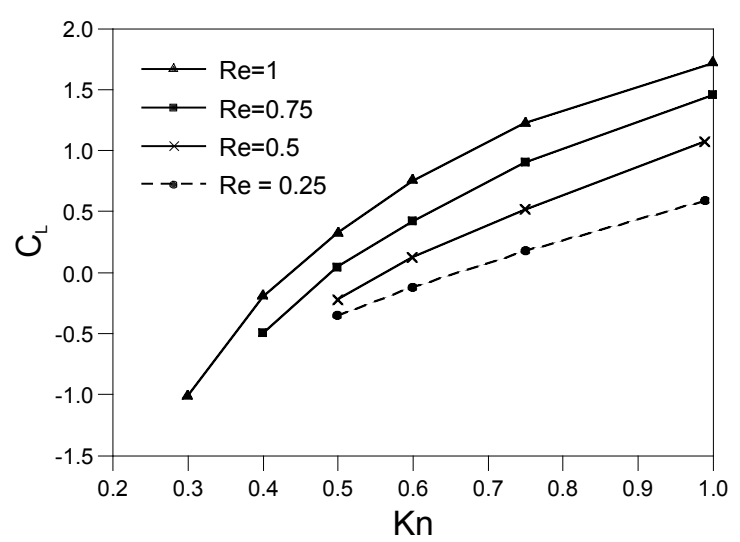

(a)

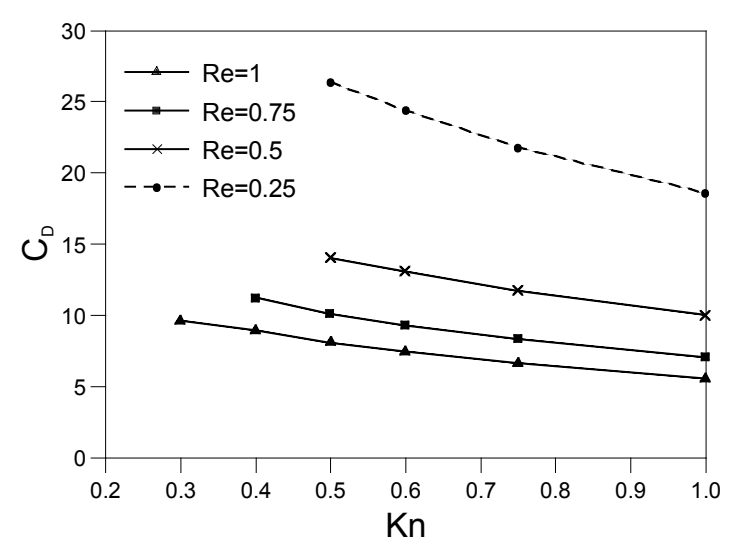

(b)

FIGURE 2. Variation of (a) lift and (b) drag coefficient as a function of $K n$ for various values of $R e$ at $W=2$ 
Figure 2(a) shows that $C_{L}$ is negative, i.e. the lift force is in the conventional Magnus direction, in the early transition regime. However, as the flow becomes more rarefied, the lift coefficient changes sign indicating the occurrence of the inverse Magnus effect. It is interesting to note that the Reynolds number significantly affects the magnitude of the lift force. The lift inversion or transition point, where the lift force is zero, occurs at lower Knudsen numbers as the Reynolds number is increased. The variation of the drag coefficient with Knudsen number is presented in Fig. 2(b). It can be seen that for a constant value of Reynolds number, the drag coefficient decreases as the flow becomes more rarefied.

To assess the impact of the Reynolds number on the inverse Magnus effect, Fig. 3 presents the coefficients of lift and drag as a function of Reynolds number. The lift variation in Fig. 3(a) demonstrates that, for relatively low values of $\mathrm{Kn}$, lift inversion will occur simply by increasing the Reynolds number. As the Knudsen number increases further, the lift coefficient is positive irrespective of the Reynolds number considered. We have also studied the impact of the Reynolds number on the drag coefficient. Figure 3(b) shows that the drag coefficient decreases as the Reynolds number is increased.

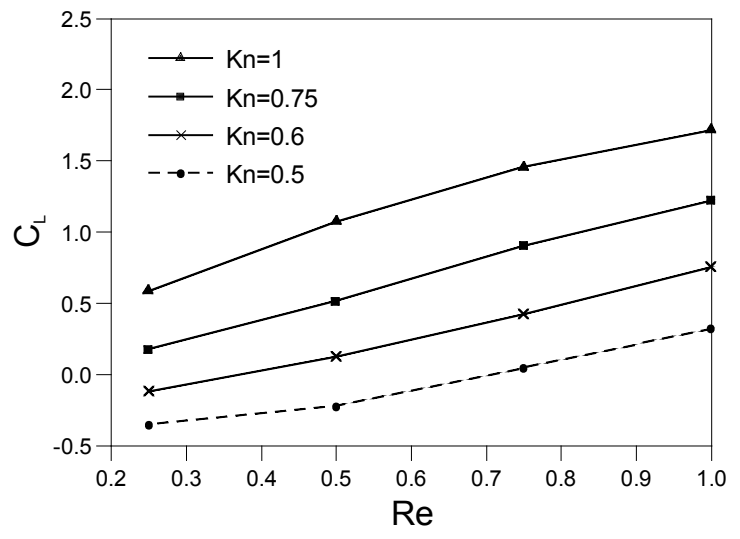

(a)

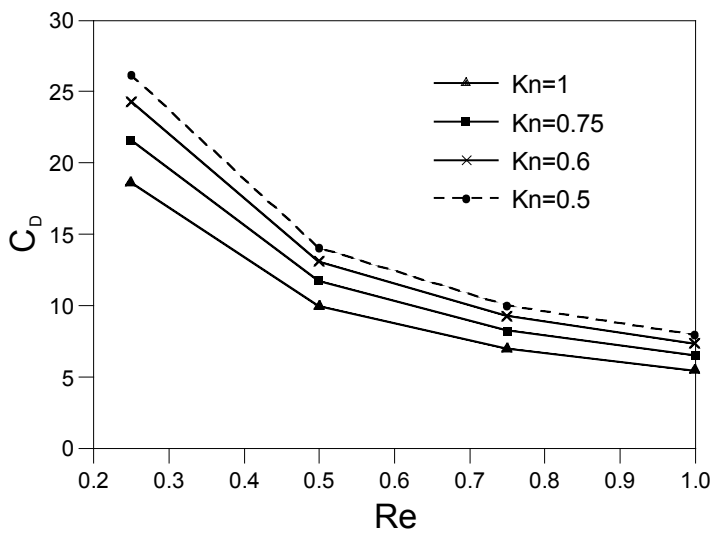

(b)

FIGURE 3. Variation of (a) lift and (b) drag coefficient as a function of $R e$ for various values of $K n$ at $W=2$

To investigate the inverse Magnus effect in more detail, it is informative to separate the lift coefficient into its pressure and viscous components. The pressure component of the lift force is evaluated from the normal momentum fluxes of the incident and reflected molecules that collide with the wall of the cylinder, whereas the viscous component of lift is evaluated from the corresponding tangential momentum fluxes acting along the wall. Since the cylinder surface is curved, the total lift force is composed of contributions from both the pressure and viscous forces.

The variations of the pressure and viscous components of the lift coefficient are presented in Fig. 4(a). The pressure component of the lift coefficient, $C_{L, p}$, is negative for all Knudsen numbers, demonstrating that the pressure always contributes to a lift force in the conventional Magnus direction. Conversely, the viscous component of the lift coefficient, $C_{L, v}$, is positive, indicating that the viscous lift is in the opposite direction to the conventional Magnus force. Additionally, it can be seen that the magnitude of $C_{L, v}$ increases with $K n$ whereas the magnitude of $C_{L, p}$ decreases as $K n$ is increased. The inverse Magnus effect can therefore be explained in terms of the increased role of the viscous contribution and the reduced role of the pressure contribution at higher degrees of rarefaction.

It is also useful to consider the pressure and viscous components of the drag coefficient. Figure 4(b) shows that both the pressure and viscous components decrease as the flow becomes more rarefied. It can also be seen that the pressure component of the drag coefficient, $C_{D, p}$, dominates the viscous component, $C_{D, v}$. 


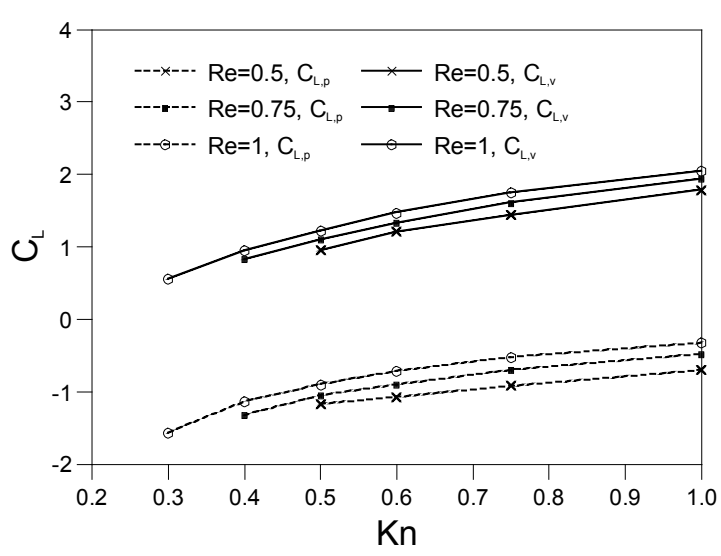

(a)

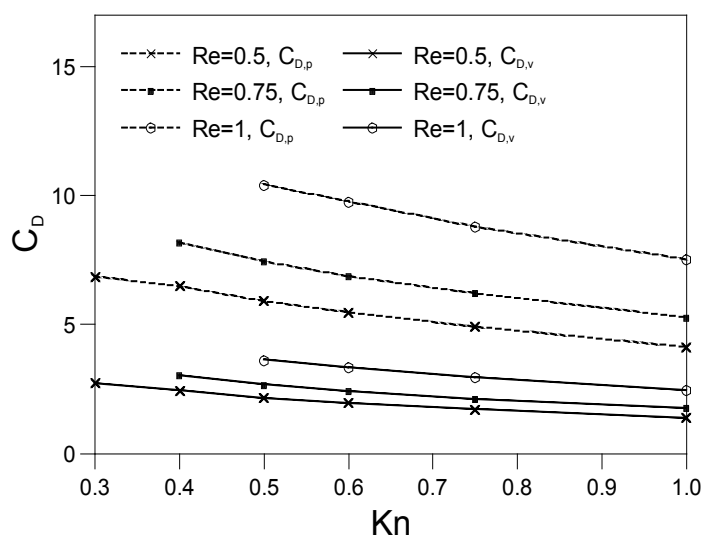

(b)

FIGURE 4. Variation of the pressure and viscous components of a) lift and b) drag coefficient as a function of $K n$ for various values of $R e$ at $W=2$

\section{Wall Temperature Sensitivity Study}

The effect of the wall temperature, $T_{w}$, on the computed aerodynamic coefficients has been assessed by performing a wall temperature sensitivity study. The cylinder wall temperature for the baseline case is assumed to be $T_{w}=273 \mathrm{~K}$ and six different wall temperatures are considered for the comparative study, i.e. $0.8 T_{w}, 0.9 T_{w}, T_{w}$, $1.1 T_{w}, 1.2 T_{w}$ and $1.4 T_{w}$. The Reynolds number was assumed to be $R e=1$ and the freestream temperature at the inflow was again specified to be $T_{\infty}=273 \mathrm{~K}$.

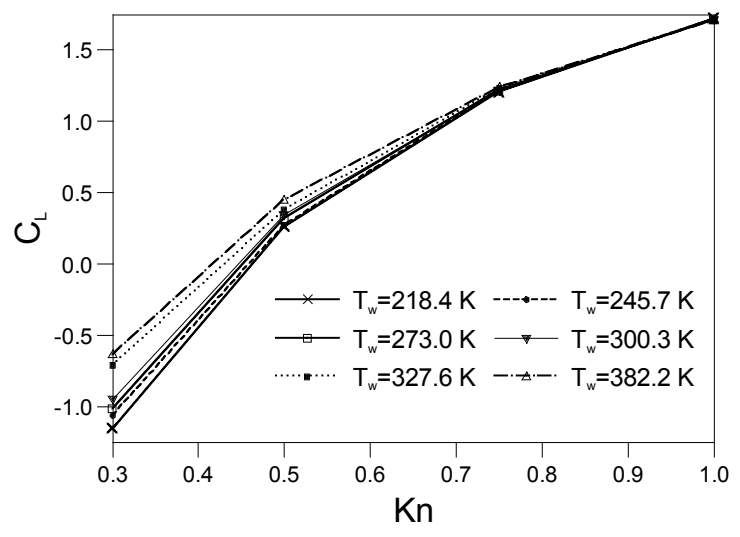

(a)

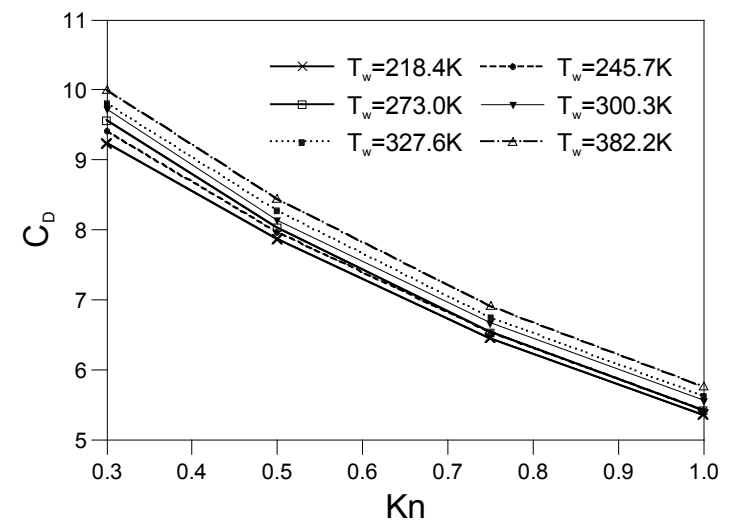

(b)

FIGURE 5. Variation of (a) lift and (b) drag coefficient as a function of $K n$ for various values of wall temperature

The variations in the lift and drag coefficients as a function of Knudsen number are shown in Fig. 5 for the six values of $T_{w}$ considered. From the lift variation plot shown in Fig. 5(a), it can be seen that the inverse Magnus effect occurs for all cases of $T_{w}$ considered. However, the lift inversion point, where the lift force is zero, is particularly sensitive to $T_{w}$, with lift inversion occurring at lower values of $K n$ as the cylinder wall temperature is increased. From Fig. 5(b), it can be seen that the drag coefficient increases as the wall temperature is increased. 
Figure 6 presents the variations of the pressure and viscous components of the lift coefficient for the various wall temperatures considered. The pressure component of the lift coefficient, $C_{L, p}$, is much more sensitive to the cylinder wall temperature than the viscous component, $C_{L, v}$. In particular, the pressure component of the lift coefficient is especially sensitive to the wall temperature in the early transition regime. However, with further increases in flow rarefaction, the pressure and viscous components of the lift coefficient become less sensitive to changes in the wall temperature. The pressure component of the lift coefficient is found to be in the conventional Magnus direction for all the cases of $K n$ and $T_{w}$ considered. In contrast, the viscous component of the lift coefficient is always positive, signifying that the viscous component is in the opposite direction to the conventional Magnus force.

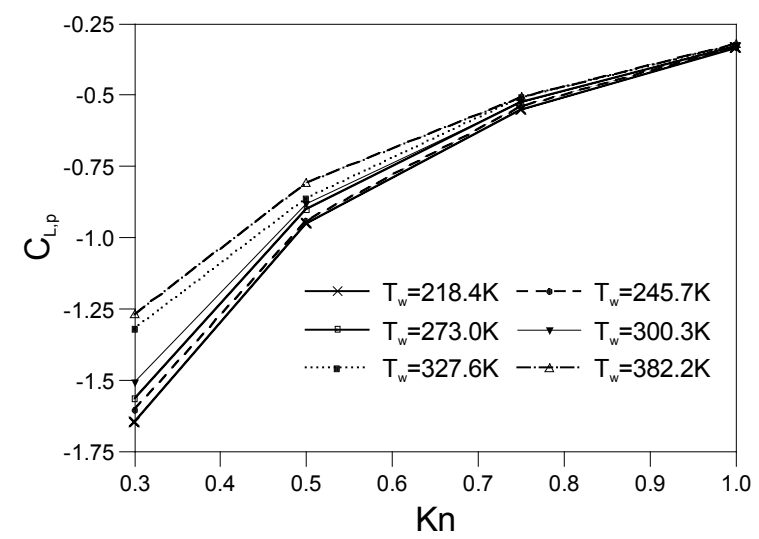

(a)

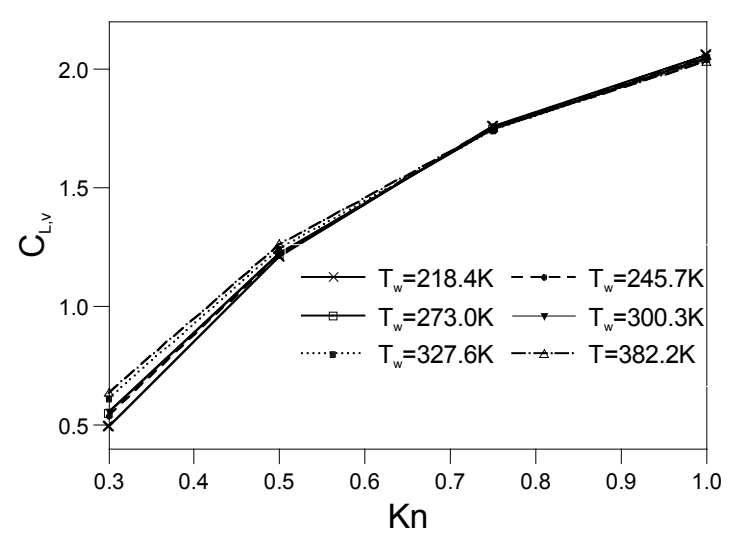

(b)

FIGURE 6. Variation of (a) pressure and (b) viscous component of the lift coefficient as a function of $K n$ for various values of wall temperature

The sensitivity of the wall temperature on the pressure and viscous components of the drag coefficient are presented in Fig. 7. The pressure component of the drag coefficient is again more sensitive to the wall temperature than the viscous component. Interestingly, the pressure and viscous components of drag follow opposite trends when the wall temperature is varied. The pressure component of the drag coefficient, $C_{D, p}$, increases with $T_{w}$ which is consistent with the variation in the total drag shown in Fig. 5(b). However, the viscous component of the drag coefficient, $C_{D, v}$, is found to decrease as the wall temperature is increased.

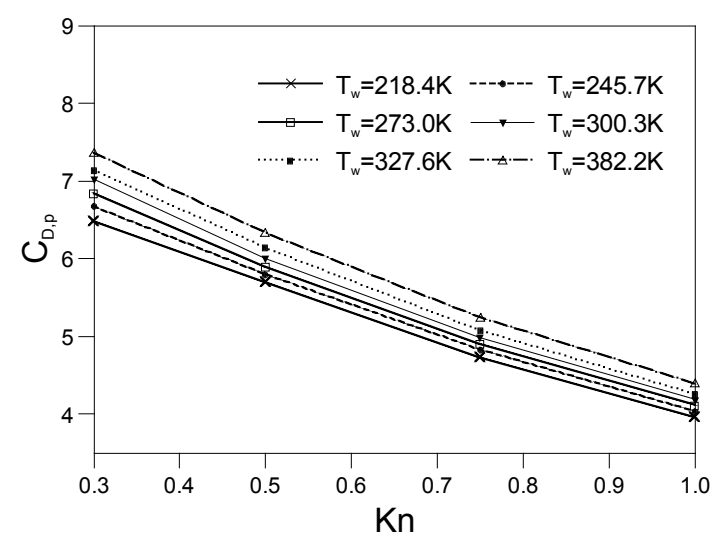

(a)

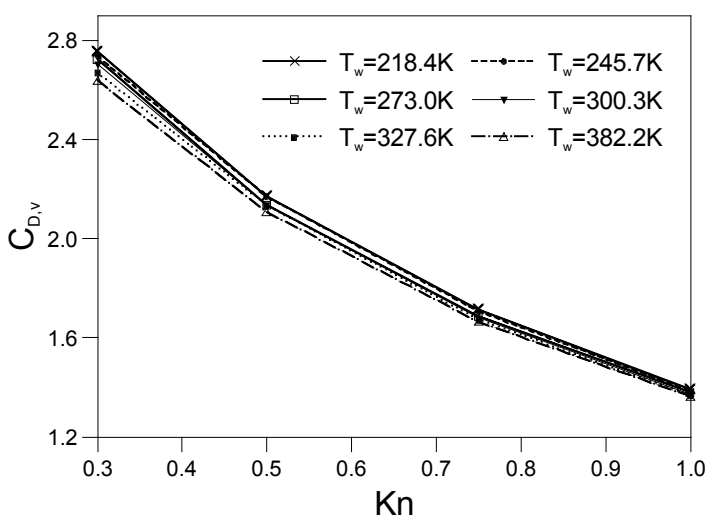

(b)

FIGURE 7. Variation of (a) pressure and (b) viscous component of the drag coefficient as a function of $K n$ for various values of wall temperature 


\section{SUMMARY AND CONCLUSIONS}

The aerodynamic characteristics of rarefied subsonic flow past a rotating cylinder have been investigated using the direct simulation Monte Carlo method. The study has focused on the transition flow regime where an inverse Magnus effect involving a change in the direction of the lift force can be observed. Both the Reynolds number and the Knudsen number have a significant effect on the magnitude of the lift coefficient and also the inversion point where the generated lift force is zero. The lift inversion point occurs at lower Knudsen numbers as the Reynolds number is increased. A wall temperature sensitivity study has also been carried out which demonstrates that the magnitude of both the lift and drag coefficients are significantly influenced by the temperature of the cylinder, particularly in the early transition regime. The inverse Magnus effect occurs for all wall temperatures considered and can be explained in terms of the increased role of the viscous contribution to the lift force combined with the reduced role of the pressure component of the lift force as the flow becomes more rarefied. A detailed investigation of the underlying physics of the inverse Magnus effect will be the subject of future studies.

\section{ACKNOWLEDGMENTS}

This work was supported through the United Kingdom Engineering and Physical Sciences Research Council under grants EP/K038427/1 - "The First Open Source Software for Non-Continuum Flows in Engineering" and EP/N016602/1 - "Nano-Engineered Flow Technologies: Simulation for Design across Scale and Phase". The authors would also like to thank Dr. Tom Scanlon of the University of Strathclyde for providing an updated version of the dsmcFoam code.

\section{REFERENCES}

1. L. Prandtl and O. G. Tietjens, Applied Hydro- and Aeromechanics (Dover, New York, 1957, 1st ed.).

2. H. J. Lugt, Vortex Flow in Nature and Technology (John Wiley \& Sons, New York, 1983).

3. W. M. Swanson, J. Basic Eng. 83, 461-470 (1961).

4. B. Robins, New Principles of Gunnery, edited by C. Hutton (F. Wingrave, London, 1805; originally published in 1742).

5. G. Magnus, Abhandlung der Akademie der Wissenschaftern, Berlin, Germany, 1852, pp. 1-24. English translation: "On the deviation of projectiles; and on a remarkable phenomenon of rotating bodies," in Scientific Memoirs, Selected from the Transactions of Foreign Academies of Science, and from Foreign Journals Natural Philosophy, edited by J. Tyndall and W. Francis (Taylor and Francis, London, 1853), pp. 210-231.

6. I. Newton, Phil. Trans. Roy. Soc. 6, No. 69-80, 3075-3087 (1671).

7. L. Prandtl, Die Naturwissenschaften 13, 93-108 (1925). English translation: "Application of the 'Magnus Effect' to the wind propulsion of ships," NACA Technical Memorandum NACA-TM-367, June 1926.

8. J. Seifert, Prog. Aerosp. Sci. 55, 17-45 (2012).

9. P. T. Tokumaru and P. E. Dimotakis, J. Fluid Mech. 255, 1-10 (1993).

10. S. Mittal and B. Kumar, J. Fluid Mech. 476, 303-334 (2003).

11. G. R. Karr and S. M. Yen, "Aerodynamic properties of spinning convex bodies in a free molecule flow," in Proceedings of the Seventh International Symposium on Rarefied Gas Dynamics, edited by D. Dini et al., (Editrice Tecnico Scientifica, Pisa, Italy, 1971), Vol. 1, pp. 339-346.

12. C. T. Wang, AIAA J. 10, $713-714$ (1972).

13. S. G. Ivanov and A. M. Yanshin, Fluid Dyn. 15, 449-453 (1980).

14. K. I. Borg, L. H. Söderholm, and H. Essén, Phys. Fluids 15, 736-741 (2003).

15. P. D. Weidman and A. Herczynski, Phys. Fluids 16, L9-L12 (2004).

16. V. V. Riabov, J. Spacecraft Rockets 36, 486-488 (1999).

17. G. A. Bird, Molecular Gas Dynamics and the Direct Simulation of Gas Flows (Clarendon Press, Oxford, 1994).

18. B. John, X. J. Gu, R. W. Barber, and D. R. Emerson, "High speed aerodynamic characteristics of rarefied flow past stationary and rotating cylinders," in 20th AIAA International Space Planes and Hypersonic Systems and Technologies Conference, 6-9 July 2015, Glasgow, Scotland. (Paper No: AIAA 2015-3511).

19. B. John, X. J. Gu, R. W. Barber, and D. R. Emerson, AIAA J. 54, 1670-1681 (2016). 
20. N. G. Hadjiconstantinou, A. L. Garcia, M. Z. Bazant, and G. He, J. Comput. Phys. 187, 274-297 (2003).

21. T. J. Scanlon, E. Roohi, C. White, M. Darbandi, and J. M. Reese, Comput. Fluids 39, 2078-2089 (2010).

22. OpenFOAM: The Open Source CFD Toolbox - User Guide, Version 2.3.1, 2014.

23. T. J. Scanlon, C. White, M. K. Borg, R. C. Palharini, E. Farbar, I. D. Boyd, J. M. Reese, and R. E. Brown, AIAA J. 53, 1670-1680 (2015).

24. R. C. Palharini, C. White, T. J. Scanlon, R. E. Brown, M. K. Borg, and J. M. Reese, Comput. Fluids 120, 140157 (2015).

25. G. J. Maslach and S. A. Schaaf, Phys. Fluids 6, 315-321 (1963).

26. S. A. Schaaf and P. L. Chambré, Flow of Rarefied Gases (Princeton University Press, Princeton, NJ, 1961).

27. Q. Sun and I. D. Boyd, AIAA J. 42, 1066-1072 (2004).

28. C. Hirsch, Numerical Computation of Internal and External Flows - Volume 2: Computational Methods for Inviscid and Viscous Flows (John Wiley \& Sons, Chichester, West Sussex, England, 1990).

29. F. J. Alexander, A. L. Garcia, and B. J. Alder, Phys. Fluids 10, 1540-1542 (1998).

30. N. G. Hadjiconstantinou, Phys. Fluids 12, 2634-2638 (2000). 